\title{
A Web of Science based bibliometric reconnaissance of the Himalaya- Karakoram-Hindukush-Tibet region with focus on field sciences
}

\author{
Pitambar Gautam \\ Creative Research Institution, Hokkaido University, N21W10, Kita-Ku, \\ Sapporo, Hokkaido 001-0021, Japan \\ Email: pitambargautam@yahoo.co.jp
}

\begin{abstract}
A bibliometric survey of the Himalaya-Karakoram-Hindukush-Tibet (HKHT) region, the largest mountain system on Earth, for research publications recorded in the Web of Science (WOS) during 1901-2018 revealed 46,746 citable documents (articles, reviews, letters and notes) showing exponential growth mainly after 1980s. The HKHT publications that cover 244 WOS subject categories (SCs) have been used to determine the relative shares by HKHT units, countries, research organizations and publication sources. Nine WOS SCs related to "earth, environmental and agricultural sciences" exhibit highest shares $(22.6 \%$ to $3.2 \%$ of the total) by the whole counting method. Further analysis of the 1994-2018 subset related to 4 broader disciplinary classes (Geosciences, Environmental Sciences \& Technologies, Agricultural Sciences, and Ecological Sciences) attributed to "field sciences" with particular emphasis on the high impact (TOP10\% globally by citation) documents enables to capture the most prolific, representative (both in space and time) and impactful research. This study identifies the prolific countries, institutions, journals, etc. characterizing the cross-disciplinary research transcending national boundaries and involving international teams. Science mapping of high impact publications (4,561 documents) using the co-occurrence of keywords restricted to noun phrases reveals six prominent clusters that reflect the prolific and high impact research themes in field science for the whole HKHT region: five of them related to earth and environmental sciences (climate change including monsoon regime, tectonic evolution of the Himalaya-Tibet orogen, India-Asia collision and associated crustal phenomena, activities on major thrusts, channel flows and inverted metamorphism), and one contrasting theme concerning the genetic diversity of plants mainly of medicinal values.
\end{abstract}

Keywords: Bibliometrics; Himalaya; Tibet; Science mapping; Field sciences

Received: 15 March, 2021 Received in revised form: 29 May, 2021 Accepted: 3 June, 2021

\section{INTRODUCTION}

The Himalaya-Karakoram-Hindukush-Tibet (HKHT) region (Fig. 1) of elevated topography resulting from the India-Asia collision and with diverse geographical, geological, biological and environmental/climatic conditions serves as a natural laboratory for the global research community. Sporadic research, since the early-mid 20th century, gained momentum after the initiation of the first Himalaya-KarakoramTibet (HKT) Workshop in the mid 1980s, and then continued at different global venues annually (e.g., Gautam et al., 2005). The Hindukush Himalayan Monitoring and Assessment Programme (HIMAP) dedicated to monitoring and assessment of major issues such as climate change, biodiversity, energy, cryosphere (frozen water), water, food security, air pollution, disaster and resilience, poverty, adaptation, and gender and migration further promoted the study of the region (Wester et al., 2019). In this context, there is a strong need of a comprehensive bibliometric survey of the vast amount of research output by researchers from a large number of countries in the past. To partially address such a need, this study performs, at first, a preliminary but comprehensive bibliometric survey covering over 100 years of the history of research publications on the HKHT region. After that, it attempts a focused study on the so-called field sciences (Agricultural Sciences; Ecological Sciences; Geosciences; Environment Sciences \& Technologies - selected categories used by Rafols (2014) for science mapping) found to be prolific in the HKHT region.

Bibliometric analysis for the entire region as an integrated orogenic system and its constituents is believed to help to accurately understand and quantify the research entities (countries, universities and research institutes, governmental and nongovernmental organizations/agencies, etc.) and to identify the prolific journals used as the publication outlets. The basic assumption behind this study is that science mapping of the whole dataset helps to identify the temporal trends and major research topics, while a focused study of the high-impact (e.g., top $10 \%$ by citation, normalized for the subject categories) papers reveals the topics of established research strengths, while the text mining enables further to detect the emerging topics. While the collision tectonics and related geological phenomena, biodiversity, environmental/climatic processes closely associated with mountain uplift, initiation/intensification of the 


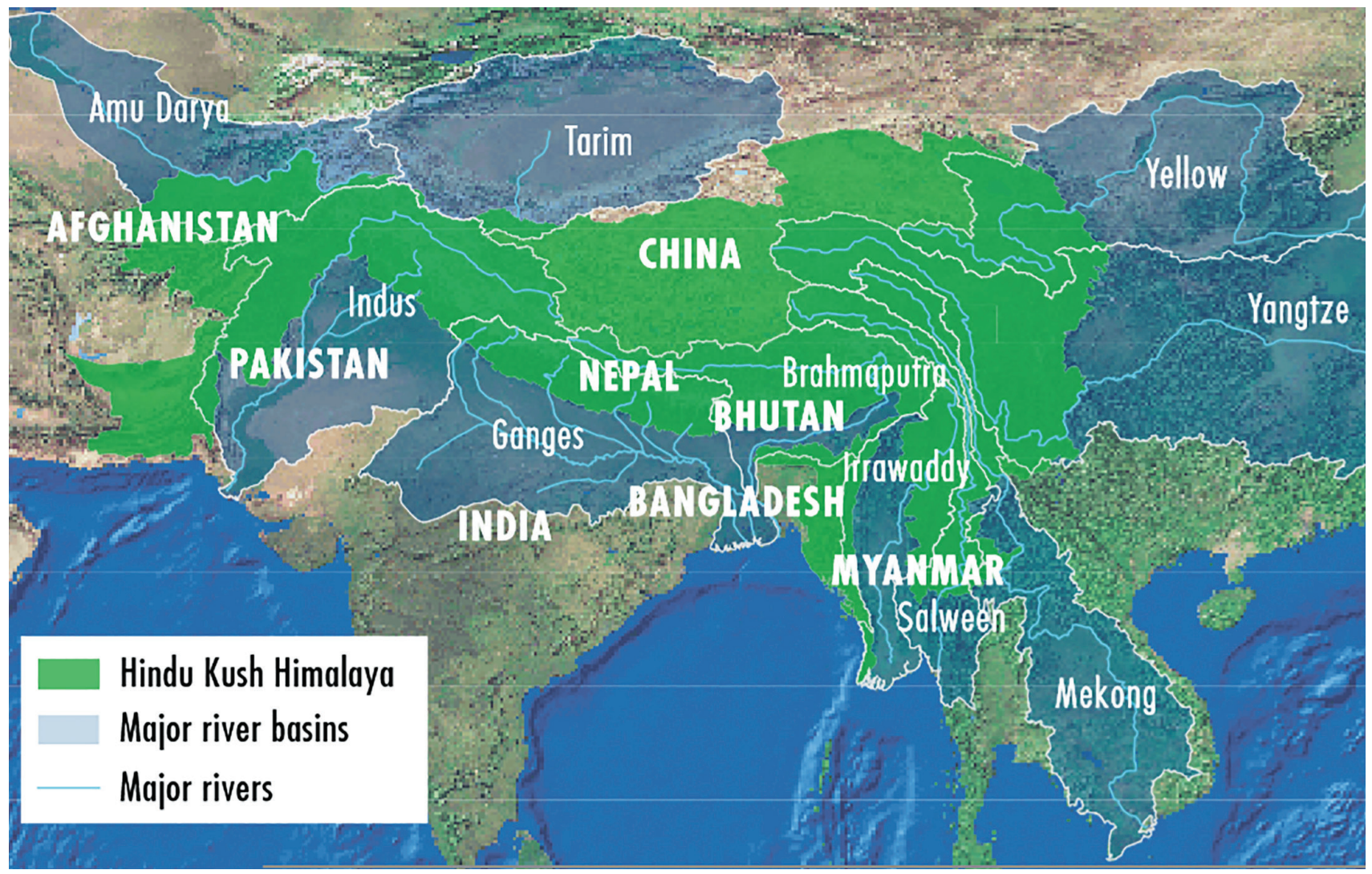

Fig. 1: Part of the world map showing the "Hindu Kush Himalaya" region and associated major river basins in Asia (after Wester et al. 2019) that include the mountain ranges covered by the Himalaya-KarakoramHindukush-Tibet (HKHT) region discussed in this study.

monsoon regime have been the research targets in the region for several decades, new topics of both scientific and societal relevance related with the disastrous earthquakes, glacier lakes outburst floods, extreme precipitation, landslides, and global warming related to climate change phenomena seem to have received much attention during the recent 5-15 years period.

In the context of increasing use of the bibliometric/ scientometric methods to understand earth and environmental processes (e.g., studies on earthquakes by Liu et al. (2012) and on glacial lake outburst floods by Emmer (2018)) and the yet restricted nature of such studies within the HKHT region (e.g., Indian Himalayas: Gupta and Gupta, 2014; Prasad et al., 2016; Tibetan Plateau: Wang, 2017 (in Chinese); Himalayan plants: Hao et al., 2019; Ecosystem services: Kandel et al., 2021), this study aims at comprehensive understanding of the entire HKHT system with its constituents that resemble in many respects such as topography, geology, environment/ climate, biodiversity, agricultural practices. The aim of this study is to get a preliminary bibliometric/ scientometric overview, on the internationally contributed as well as peer-reviewed research output on the HKHT region, to serve as a base for further future research with focus on the specific targets and disciplinary areas/themes.

\section{METHODOLOGY}

Research publications data related to the HKHT region were harvested from the Web of Science (WOS) Core Collection (CC) comprising 4 database modules (SCIE, SSCI A\&HCI, ESCI; managed by Clarivate Analytics) in the 'advanced search' mode. The choice of strings for topics (title, abstract, author and publisher keywords) search (TS) were as follows:

Himalayan mountain region: TS=him*lay*;

Karakoram mountain range: TS=(Kar?kor* OR Karakor*);

Hindukush mountain range: TS=((hind*kus* OR hindu-kus* OR hind-kus*) NOT "hind kusht");

Tibetan Plateau: TS $=$ (tibet* OR xizang)

At first, the 1901-2018 dataset of research publications, comprising 4 types of citable items (article, review, letter and note), was analyzed. It was followed by a focused analysis of 1994-2018 publications in field sciences, related to a group of the WOS subject categories (SCs), of relatively better coverage in WOS (Aksnes and Sivertsen, 2019). In addition, the articles and reviews with relatively high impact (top $10 \%$ by citation counts, after normalization for SCs, publication year and document type) limited to SCIE, SSCI and A\&HCI modules, known for the quality content, were also studied. The present bibliometric 
analysis and science mapping follows the commonly established approaches found in the published literature (e.g., Glänzel, 2003; van Eck and Waltman, 2010; Rafols, 2014; Gautam, 2017; Gautam, 2019) using the InCites analytical tool along with WOS managed by Clarivate Analytics (2019). Data from WOS and InCites, used for the reasons of robustness and accessibility by subscription, were retrieved from a web-based portal at Hokkaido University, mainly on May 28, 2019. Data processing and compilation was done using spreadsheets (Microsoft Excel), while VantagePoint (Search Technology, Inc.) and VOSviewer (Leiden University) were used for data cleaning, disciplinary classifications and science mapping. A science overlay map for the HKHT region was generated using the counts of WOS SCs, data on clusters based on their aggregation into 18 disciplinary classes, and the global map of science based on aggregated journal citation data in 2015 following the procedure after Leydesdorff (2021).

\section{RESULTS AND INTERPRETATION}

\section{Basic Bibliometric Indicators for the HKTH Region and its Constituents}

\section{Publication Volume and Trend}

The total number of 1901-2018 research publications on the HKHT region in WOS-indexed journals is 46,746 comprising 44,530 articles, 1,549 reviews, 348 letters and 319 notes. Documents counts by whole counting for the constituent regions were as follows: 27,465 for Tibet, 22,996 for Himalaya, 1,398 for Karakoram, and 772 for Hindukush. Tibet and
Himalaya together comprise 46,111 documents. As 4,350 documents are assigned to both regions, there is a mutual overlap of $9.4 \%$. Document counts unique to Karakoram and Hindukush were 327 (23.4\%) and $275(35.6 \%)$, respectively, implying that there is a significant overlap of publications assigned to them with Himalaya and/or Tibet. The cumulative number of documents for the HKHT region reached a level of merely $1.75 \%$ by 1980 , when the annual document count approached close to $100(0.21 \%)$. For clarity, therefore, a bi-plot of publications versus year is limited to the period between 1980 and 2018 (Fig. 2). An exponential increase in the cumulative research output is graphically evident for Himalaya and Tibet, with an initially delayed but recently accelerated trend for the latter.

\section{Share of Publications by Countries}

Table 1 is a compilation of contribution by countries/ regions to research publications during 1901-2018. Number of countries/regions engaged in publications and those producing at least 5 documents (in brackets) are as follows: HKHT: 160 (112); Tibet: 135 (83); Himalaya: 139 (97); Karakoram: 67 (36); and, Hindukush: 72 (30). When the entire HKHT region is considered, China and India top the list of contributors and they are followed by USA, Germany, England, France, Japan, Australia and Canada, which occupy the third to ninth positions. Nepal and Pakistan are in the 10th and 11th positions, respectively. Data are also given for other countries that are situated outside the HKHT region but produce more than 200 documents during the 118 yrs. period. Likewise, publications

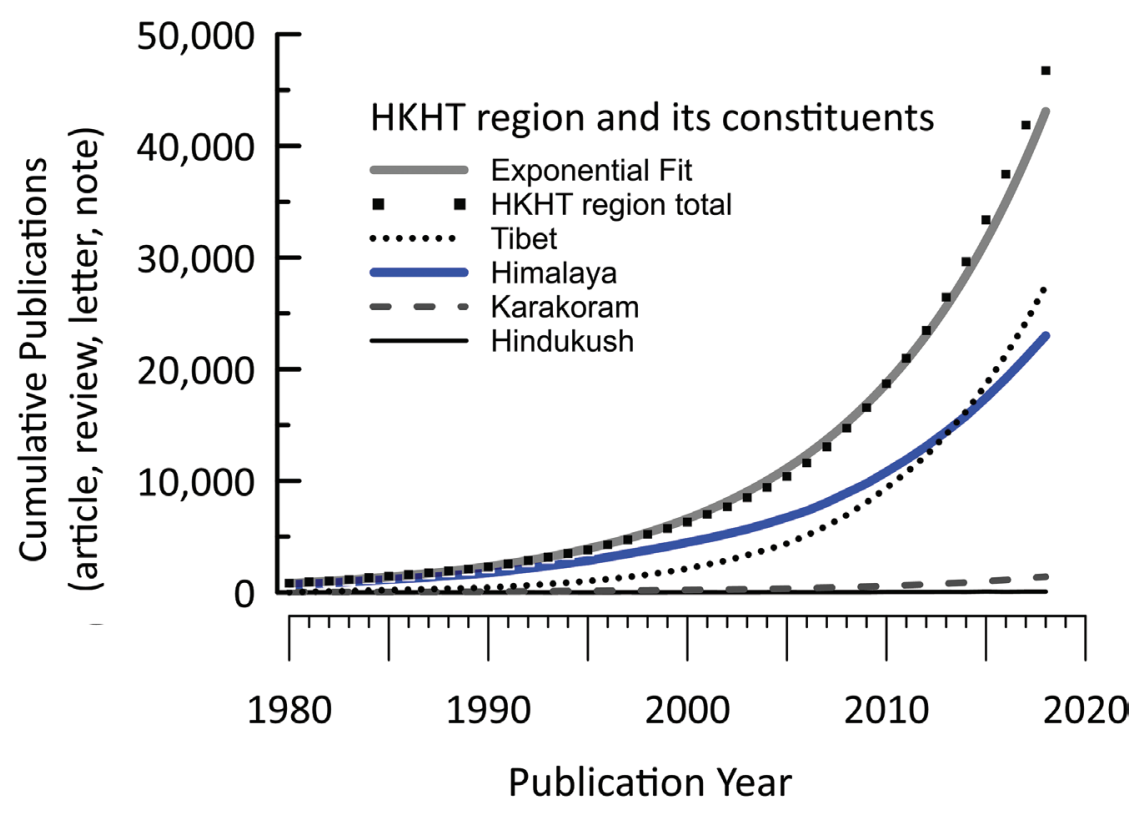

Fig. 2: Cumulative research output related to the Himalaya-Karakoram-Hindukush-Tibet (HKHT) region by the year of publication retrieved from the Web of Science database. Numbers for individual constituent were obtained by whole counting when publications were assigned to several constituent regions. 
data for countries linked to the HKHT region (Bhutan, Bangladesh, Myanmar and Afghanistan) are also listed notwithstanding with their relatively smaller shares.

\section{Major Organizations Contributing to Research Publications}

Organizations/institutions engaged in the production of the research publications were identified using the counts for items entitled "organization-enhanced" and "organizations" (more than 10,000 titles for the HKHT region) using the "analyze results" option available in WOS. Organization names required cleaning, disambiguation at several hierarchical levels, and occasionally filtering or grouping -performed manually using the spreadsheet capabilities. Because of the possible changes of the names of organizations due to restructuring or merging during the long time span (1901-2018) covered by the research publications, the difficulty of identifying the actual status of numerous organizations/institutes of large-scale public or governmental organizations/ agencies such as the science academies, research councils and affiliated institutes, recent trend of creating new university systems by merging several to dozen or more independent universities, and an incomplete description of affiliations in papers and database, the document counts and shares may have been underestimated in several instances. For large organizations and systems like the Chinese Science Academy or Indian Institute of Technologies (IIT), the most productive members (in italics) are also identified in the respective Tables. Although the approach used here to discriminate the organizations has some subjective element, it is believed to be adequate to capture the major contributors aimed by this reconnaissance study.

Breakdown of 1901-2018 documents at the level of research organizations is presented in Table 2. In the datasets related to Tibet, Himalaya and Karakoram, there is an overall predominance of the Chinese Academy of Sciences (with 4 topmost contributing members identified in the list). The International Center for Integrated Mountain Development (ICIMOD) based in Nepal is the top contributor to the Hindukush publications. For Tibet, the only non-Chinese organizations (systems) with relatively high shares $(>1 \%$ documents) are CNRS (France), University of California System, University of Colorado System, and Helmholtz Association.

For Himalaya, besides the Chinese Academy of Sciences and its major members that clearly have the predominant share, several singular institutions from India (Wadia Institute of Himalayan Geology, Kumaon University, GB Pant Institute of Himalayan
Environment, IIT Roorkee, HNB Garhwal University, and National Geophysical Research Institute) and from Nepal (Tribhuvan University) show $>1 \%$ share. For Karakoram, additional institutions such as Université Grenoble Alpes, University of Milan, University of Oxford, Russian Academy of Sciences, University of London, and ICIMOD occupy the higher ranks by the publication shares. The Hindukush-related publications, despite a low volume, show a large international diversity of prolific contributors such as Indian Space Research Organization, University of Kashmir, University of Hong Kong, Université de Paris, and University of Cambridge, which are not so prominent in other regions.

\section{Share of Publications by WOS Subject Categories and 18 Disciplinary Classes}

The HKHT dataset has a high share $(99.5 \%)$ of articles and reviews, which are commonly considered appropriate for evaluative bibliometrics. This set covers 244 WOS SCs comprising 254 titles in total. Table 3 lists top 10 productive categories characterizing the whole region. Eleven SCs (percentage share by whole counting) with more than 1000 counts top the list: Geosciences, Multidisciplinary (22.6\%); Geochemistry \& Geophysics (12.9\%); Environmental Sciences (9.5\%); Meteorology \& Atmospheric Sciences (8.9\%); Plant Sciences (7.2\%); Geography, Physical (6.9\%); Geology (5.1\%); Ecology (5.1\%); Water Resources (3.6\%); Genetics \& Heredity (2.7\%); and, Zoology (2.7\%). Except for Genetics \& Heredity, each of these categories belongs to one of the 4 broad disciplinary classes of field sciences scrutinized further in this paper.

Publications data for the HKHT region aggregated into 18 classes, each of which consists of several WOS SCs, are presented in Table 4 . With slightly over $50 \%$ of the documents, the most prolific class is Geosciences. Each of the next four classes (Agricultural Sciences, Ecological Sciences, Environmental Sciences \& Technologies, and Biomedical Sciences) has a documentary share of slightly over $10 \%$ and thus is moderately important for the HKHT region. The following eight classes with rather low (1-5\%) but significant shares belong to the "science, technology, engineering and medicine" (Infectious Diseases; Chemistry; Clinical Medicine; Engineering; Material Sciences) and the "social sciences \& humanities" (Economics, Politics \& Geography; Social Studies; Health \& Social Issues) areas.

\section{Science Overlay Map for the HKHT Region}

Figure 3 presents a VOSviewer generated science overlay map for the HKHT region based on the publications covered by WOS SCs, with their counts being proportional to the size of the nodes and their 
A Web of Science based bibliometric reconnaissance of the Himalaya-Karakoram-Hindukush-Tibet region

Table 1: Countries contributing to research publications (1901-2018) on HKHT region.

\begin{tabular}{|c|c|c|c|c|c|c|c|}
\hline \multirow{2}{*}{ Rank } & \multirow{2}{*}{$\begin{array}{l}\text { Country/ } \\
\text { Region }\end{array}$} & \multicolumn{2}{|c|}{ HKTH region } & \multirow{2}{*}{$\begin{array}{l}\text { Tibet } \\
\text { Ndoc }\end{array}$} & \multirow{2}{*}{$\begin{array}{c}\text { Himalaya } \\
\text { Ndoc }\end{array}$} & \multirow{2}{*}{$\begin{array}{c}\text { Karakoram } \\
\text { Ndoc } \\
\end{array}$} & \multirow{2}{*}{$\begin{array}{c}\text { Hindu Kush } \\
\text { Ndoc }\end{array}$} \\
\hline & & $\%$ share & Ndoc & & & & \\
\hline 1 & PEOPLES R CHINA* & 46.93 & 21940 & 20412 & 3629 & 279 & 104 \\
\hline 2 & INDIA* & 23.41 & 10942 & 1008 & 10522 & 205 & 107 \\
\hline 3 & USA & 19.02 & 8893 & 6181 & 3969 & 320 & 154 \\
\hline 4 & GERMANY & 5.74 & 2684 & 1714 & 1299 & 186 & 92 \\
\hline 5 & ENGLAND & 5.57 & 2605 & 1518 & 1441 & 169 & 70 \\
\hline 6 & FRANCE & 4.19 & 1959 & 1118 & 1142 & 117 & 55 \\
\hline 7 & JAPAN & 3.89 & 1820 & 1222 & 764 & 41 & 21 \\
\hline 8 & AUSTRALIA & 3.29 & 1538 & 1038 & 665 & 63 & 15 \\
\hline 9 & CANADA & 3.2 & 1498 & 941 & 788 & 107 & 24 \\
\hline 10 & NEPAL* & 2.3 & 1077 & 265 & 987 & 46 & 94 \\
\hline 11 & PAKISTAN* & 2.22 & 1039 & 159 & 832 & 198 & 154 \\
\hline 12 & SWITZERLAND & 2.16 & 1011 & 514 & 641 & 80 & 25 \\
\hline 13 & ITALY & 1.94 & 909 & 439 & 602 & 110 & 39 \\
\hline 14 & NETHERLANDS & 1.42 & 665 & 413 & 313 & 41 & 23 \\
\hline 15 & RUSSIA & 1.29 & 604 & 343 & 294 & 28 & 40 \\
\hline 16 & SCOTLAND & 1.09 & 510 & 252 & 320 & 41 & 7 \\
\hline 17 & NORWAY & 1.02 & 478 & 216 & 303 & 21 & 14 \\
\hline 18 & TAIWAN & 1.01 & 472 & 368 & 166 & 10 & 2 \\
\hline 19 & SWEDEN & 0.95 & 445 & 309 & 192 & 11 & 3 \\
\hline 20 & AUSTRIA & 0.93 & 434 & 220 & 264 & 26 & 20 \\
\hline 21 & SOUTH KOREA & 0.74 & 347 & 217 & 168 & 17 & 3 \\
\hline 22 & SPAIN & 0.74 & 346 & 209 & 172 & 10 & 8 \\
\hline 23 & NEW ZEALAND & 0.67 & 311 & 163 & 180 & 27 & 4 \\
\hline 24 & IRAN & 0.53 & 248 & 79 & 169 & 6 & 15 \\
\hline 25 & FINLAND & 0.5 & 235 & 177 & 97 & 5 & 4 \\
\hline 26 & TURKEY & 0.5 & 235 & 75 & 160 & 5 & 8 \\
\hline 27 & BELGIUM & 0.49 & 230 & 125 & 113 & 5 & 2 \\
\hline 28 & POLAND & 0.48 & 226 & 105 & 128 & 1 & 3 \\
\hline 29 & CZECH REPUBLIC & 0.47 & 218 & 98 & 146 & 12 & 7 \\
\hline 30 & DENMARK & 0.44 & 208 & 123 & 103 & 5 & 5 \\
\hline 31 & WALES & 0.41 & 190 & 71 & 128 & 16 & 3 \\
\hline 32 & THAILAND & 0.35 & 162 & 67 & 104 & 4 & 4 \\
\hline 33 & SINGAPORE & 0.32 & 148 & 94 & 76 & 6 & 0 \\
\hline 34 & SOUTH AFRICA & 0.3 & 141 & 47 & 96 & 5 & 4 \\
\hline 35 & BHUTAN* & 0.29 & 137 & 30 & 131 & 0 & 3 \\
\hline 36 & ISRAEL & 0.25 & 116 & 85 & 38 & 1 & 1 \\
\hline 37 & SAUDI ARABIA & 0.22 & 101 & 33 & 70 & 7 & 6 \\
\hline 38 & BRAZIL & 0.21 & 100 & 50 & 57 & 2 & 1 \\
\hline 39 & IRELAND & 0.21 & 100 & 78 & 39 & 5 & 3 \\
\hline 40 & VIETNAM & 0.19 & 89 & 48 & 50 & 3 & 1 \\
\hline 41 & MEXICO & 0.19 & 89 & 31 & 60 & 0 & 2 \\
\hline 42 & BANGLADESH* & 0.19 & 89 & 31 & 70 & 1 & 4 \\
\hline 43 & GREECE & 0.19 & 87 & 38 & 46 & 3 & 13 \\
\hline 44 & HUNGARY & 0.18 & 84 & 50 & 47 & 2 & 0 \\
\hline 45 & MALAYSIA & 0.18 & 82 & 18 & 63 & 2 & 2 \\
\hline 46 & MONGOL PEO REP & 0.13 & 59 & 44 & 17 & 3 & 0 \\
\hline 47 & CHILE & 0.12 & 56 & 31 & 26 & 0 & 1 \\
\hline 48 & MYANMAR* & 0.12 & 56 & 31 & 38 & 0 & 2 \\
\hline 49 & KYRGYZSTAN & 0.12 & 55 & 34 & 24 & 3 & 10 \\
\hline 50 & ARGENTINA & 0.11 & 51 & 20 & 30 & 5 & 0 \\
\hline 51 & PORTUGAL & 0.11 & 51 & 29 & 24 & 1 & 0 \\
\hline 79 & AFGHANISTAN* & 0.03 & 15 & 3 & 4 & 1 & 10 \\
\hline
\end{tabular}

Note: Countries producing 200 or more documents and countries related to the HKHT region (indicated by an asterisk) even if producing less are listed. Data source: WOS CC (SCIE, SSCI, A\&HCI, ESCI). Document types: article, review, letter, note. 
Table 2: Most productive organizations related to 1901-2018 publications by region.

\begin{tabular}{|c|c|c|c|c|c|c|}
\hline \multirow{2}{*}{$\begin{array}{l}\text { Organizations-Enhanced } \\
\text { Major member(s) }\end{array}$} & \multicolumn{2}{|c|}{$\begin{array}{l}\text { HKHT } \\
(46,746)\end{array}$} & $\begin{array}{c}\text { Tibet } \\
(27,465)\end{array}$ & $\begin{array}{l}\text { Himalaya } \\
(22,996)\end{array}$ & $\begin{array}{c}\text { Karakoram } \\
(1,398)\end{array}$ & $\begin{array}{l}\text { Hindukush } \\
\text { (772) }\end{array}$ \\
\hline & \multicolumn{2}{|l|}{ Ndoc } & \multicolumn{4}{|c|}{ Share of papers to total for each region (\%) } \\
\hline CHINESE ACADEMY OF SCIENCES & 11,162 & 23.88 & 37.78 & 8.26 & 12.09 & 7.64 \\
\hline UNIVERSITY OF CHINESE ACADEMY OF SCIENCES CAS & 3,199 & 6.84 & 10.77 & 2.23 & 4.22 & 2.85 \\
\hline INSTITUTE OF TIBETAN PLATEAU RESEARCH CAS & 1,745 & 3.73 & 6.09 & 1.84 & 5.01 & 2.72 \\
\hline $\begin{array}{l}\text { COLD ARID REGIONS ENVIRONM ENGINEERING RES INST } \\
\text { CAS }\end{array}$ & 1,446 & 3.09 & 5.04 & 0.84 & 1.36 & 1.30 \\
\hline INSTITUTE OF GEOLOGY GEOPHYSICS CAS & 1,072 & 2.29 & 3.66 & 1.11 & 2.22 & 0.78 \\
\hline CHINA UNIVERSITY OF GEOSCIENCES & 1,833 & 3.92 & 6.15 & 2.02 & 1.65 & 1.30 \\
\hline LANZHOU UNIVERSITY & 1,507 & 3.22 & 5.43 & 0.38 & 0.43 & 0.13 \\
\hline CHINESE ACADEMY OF GEOLOGICAL SCIENCES & 1,446 & 3.09 & 5.06 & 1.56 & 2.29 & 0.65 \\
\hline DEPARTMENT OF SCIENCE TECHNOLOGY INDIA & 1,356 & 2.90 & 0.69 & 5.70 & 4.79 & 1.04 \\
\hline WADIA INSTITUTE OF HIMALAYAN GEOLOGY & 887 & 1.90 & 0.36 & 3.81 & 3.29 & 0.52 \\
\hline BIRBAL SAHNI INSTITUTE OF PALAEOBOTANY & 246 & 0.53 & 0.18 & 0.98 & 1.36 & 0.13 \\
\hline $\begin{array}{l}\text { CENTRE NATIONAL DE LA RECHERCHE SCIENTIFIQUE } \\
\text { CNRS }\end{array}$ & 1,256 & 2.69 & 2.59 & 3.27 & 6.51 & 4.53 \\
\hline CNRS NATL INST FOR EARTH SCIENCES ASTRONOMY INSU & 367 & 0.79 & 0.87 & 0.86 & 1.57 & 0.13 \\
\hline UNIVERSITY OF CALIFORNIA SYSTEM & 1,212 & 2.59 & 3.27 & 2.46 & 4.36 & 3.24 \\
\hline UNIVERSITY OF CALIFORNIA LOS ANGELES & 342 & 0.73 & 1.07 & 0.60 & 1.29 & 0.65 \\
\hline $\begin{array}{l}\text { COUNCIL OF SCIENTIFIC INDUSTRIAL RESEARCH CSIR } \\
\text { INDIA }\end{array}$ & 1,200 & 2.57 & 0.60 & 5.00 & 1.65 & 1.94 \\
\hline $\begin{array}{l}\text { CSIR NATIONAL GEOPHYSICAL RESEARCH INSTITUTE } \\
\text { NGRI }\end{array}$ & 344 & 0.74 & 0.34 & 1.41 & 1.07 & 1.81 \\
\hline $\begin{array}{l}\text { INDIAN INSTITUTE OF TECHNOLOGY SYSTEM IIT } \\
\text { SYSTEM }\end{array}$ & 1,191 & 2.55 & 0.59 & 4.94 & 2.29 & 3.63 \\
\hline INDIAN INSTITUTE OF TECHNOLOGY IIT ROORKEE & 533 & 1.14 & 0.14 & 2.29 & 0.86 & 1.68 \\
\hline CHINA EARTHQUAKE ADMINISTRATION & 1,095 & 2.34 & 3.83 & 0.77 & 1.79 & 1.30 \\
\hline PEKING UNIVERSITY & 938 & 2.01 & 3.26 & 0.70 & 0.64 & 1.04 \\
\hline INDIAN COUNCIL OF AGRICULTURAL RESEARCH ICAR & 780 & 1.67 & 0.06 & 3.34 & 0.14 & 0.13 \\
\hline NANJING UNIVERSITY & 772 & 1.65 & 2.65 & 0.68 & 0.50 & 0.39 \\
\hline KUMAUN UNIVERSITY & 621 & 1.33 & 0.10 & 2.68 & 1.07 & 0.13 \\
\hline BEIJING NORMAL UNIVERSITY & 545 & 1.17 & 1.92 & 0.15 & 0.29 & 0.13 \\
\hline HELMHOLTZ ASSOCIATION & 508 & 1.09 & 1.41 & 0.86 & 1.50 & 3.11 \\
\hline CHINA METEOROLOGICAL ADMINISTRATION & 507 & 1.09 & 1.77 & 0.16 & 0.57 & 1.17 \\
\hline $\begin{array}{l}\text { G B PANT NATL INST HIMALAYAN ENVIRONMENT SUST } \\
\text { DEV }\end{array}$ & 506 & 1.08 & 0.06 & 2.19 & 0.14 & 0.65 \\
\hline RUSSIAN ACADEMY OF SCIENCES & 450 & 0.96 & 0.90 & 0.96 & 1.57 & 5.18 \\
\hline HEMWATI NANDAN BAHUGUNA GARHWAL UNIVERSITY & 423 & 0.91 & 0.03 & 1.84 & 0.14 & 0.26 \\
\hline SICHUAN UNIVERSITY & 417 & 0.89 & 1.46 & 1.84 & 0.07 & \\
\hline $\begin{array}{l}\text { CHINESE ACADEMY OF METEOROLOGICAL SCIENCES } \\
\text { CAMS }\end{array}$ & 407 & 0.87 & 1.45 & 0.13 & 0.14 & 0.65 \\
\hline MINISTRY OF EARTH SCIENCES MOES INDIA & 394 & 0.84 & 0.31 & 1.50 & 0.57 & 1.94 \\
\hline $\begin{array}{l}\text { NANJING UNIVERSITY OF INFORMATION SCIENCE } \\
\text { TECHNOLOGY }\end{array}$ & 386 & 0.83 & 1.35 & 0.12 & 0.29 & 0.78 \\
\hline SUN YAT SEN UNIVERSITY & 374 & 0.80 & 1.25 & 0.27 & 0.22 & 0.13 \\
\hline UNIVERSITY OF COLORADO SYSTEM & 364 & 0.78 & 1.04 & 0.62 & 0.50 & 0.78 \\
\hline UNIVERSITY OF COLORADO BOULDER & 303 & 0.65 & 0.85 & 0.55 & 0.50 & 0.65 \\
\hline TIBET UNIVERSITY & 356 & 0.76 & 1.29 & 0.14 & & \\
\hline CHENGDU UNIVERSITY OF TECHNOLOGY & 355 & 0.76 & 1.23 & 0.24 & 0.22 & \\
\hline UNIVERSITY OF LONDON & 354 & 0.76 & 0.81 & 0.79 & 1.29 & 0.39 \\
\hline UNIVERSITE GRENOBLE ALPES UGA & 343 & 0.73 & 0.60 & 1.12 & 4.01 & 2.07 \\
\hline TRIBHUVAN UNIV & 351 & 0.75 & 0.30 & 1.47 & 0.14 & 1.43 \\
\hline UNIVERSITY OF OXFORD & 339 & 0.73 & 0.79 & 0.86 & 3.22 & 1.43 \\
\hline $\begin{array}{l}\text { INT CTR FOR INTEGRATED MOUNTAIN DEVELOPMENT } \\
\text { ICIMOD* }\end{array}$ & 244 & 0.52 & 0.22 & 0.44 & 2.78 & 9.84 \\
\hline UNIVERSITY OF MILAN & 114 & 0.24 & 0.12 & 0.31 & 3.65 & 1.68 \\
\hline
\end{tabular}

Document types: article, review, letter, note indexed in WOS Core Collection. Data based on the "Analyze Results" tool in WOS. Data for ICIMOD found under several name variants were cleaned and aggregated manually. 
Table 3: Top 10 WOS subject categories related to 1901-2018 publications by region.

\begin{tabular}{|c|c|c|c|c|c|c|c|}
\hline \multirow{2}{*}{ S. No. } & \multirow{2}{*}{ Web of Science Category } & \multicolumn{2}{|c|}{ HKTH region } & \multirow{2}{*}{$\begin{array}{l}\text { Tibet } \\
\text { Ndoc }\end{array}$} & \multirow{2}{*}{$\begin{array}{c}\text { Himalaya } \\
\text { Ndoc } \\
\end{array}$} & \multirow{2}{*}{$\begin{array}{c}\text { Karakoram } \\
\text { Ndoc }\end{array}$} & \multirow{2}{*}{$\begin{array}{c}\text { Hindu Kush } \\
\text { Ndoc }\end{array}$} \\
\hline & & $\%$ share & Ndoc & & & & \\
\hline 1 & $\begin{array}{l}\text { Geosciences, } \\
\text { Multidisciplinary }\end{array}$ & 22.64 & 10581 & 6298 & 5507 & 580 & 202 \\
\hline 2 & $\begin{array}{l}\text { Geochemistry \& } \\
\text { Geophysics }\end{array}$ & 12.94 & 6047 & 4216 & 2914 & 250 & 172 \\
\hline 3 & Environmental Sciences & 9.47 & 4429 & 2587 & 2110 & 126 & 97 \\
\hline 4 & $\begin{array}{l}\text { Meteorology \& } \\
\text { Atmospheric Sciences }\end{array}$ & 8.84 & 4131 & 3101 & 1371 & 101 & 68 \\
\hline 5 & Plant Sciences & 7.21 & 3370 & 1295 & 2203 & 31 & 37 \\
\hline 6 & Geography, Physical & 6.94 & 3242 & 2073 & 1496 & 263 & 92 \\
\hline 7 & Geology & 5.09 & 2378 & 1531 & 1248 & 98 & 25 \\
\hline 8 & Ecology & 5.03 & 2349 & 1342 & 1111 & 29 & 26 \\
\hline 9 & Water Resources & 3.62 & 1694 & 753 & 1010 & 89 & 54 \\
\hline 10 & Genetics \& Heredity & 2.74 & 1281 & 876 & 486 & 3 & 11 \\
\hline
\end{tabular}

Document types: article, review, letter, note; Ndoc $=$ No. of documents by whole counting method. As one-third of the HKHT-related documents are assigned to 2-6 subject categories based on the source journal, the sum of documents of all categories by whole counting $(68,618)$ is exaggerated by a factor of 1.47 of the actual count $(46,746)$.

Table 4: Breakdown of the HKHT research publications (1901-2018) into 18 disciplinary classes.

\begin{tabular}{|c|c|c|c|c|c|c|c|}
\hline \multirow{2}{*}{ S. No. } & \multirow{2}{*}{$\begin{array}{l}\text { WOS } 18 \text { disciplinary } \\
\text { classes* }\end{array}$} & \multicolumn{2}{|c|}{ HKTH region } & \multirow{2}{*}{$\begin{array}{l}\text { Tibet } \\
\text { Ndoc }\end{array}$} & \multirow{2}{*}{$\frac{\text { Himalaya }}{\text { Ndoc }}$} & \multirow{2}{*}{$\begin{array}{c}\text { Karakoram } \\
\text { Ndoc }\end{array}$} & \multirow{2}{*}{$\begin{array}{c}\text { Hindu Kush } \\
\text { Ndoc }\end{array}$} \\
\hline & & $\%$ share & Ndoc & & & & \\
\hline 1 & Geosciences & 52.38 & 24484 & 15904 & 11660 & 1094 & 496 \\
\hline 2 & Agricultural Sciences & 13.02 & 6085 & 2491 & 3754 & 46 & 52 \\
\hline 3 & Ecological Sciences & 12.60 & 5888 & 3265 & 2924 & 57 & 68 \\
\hline 4 & $\begin{array}{l}\text { Environment Sciences \& } \\
\text { Technologies }\end{array}$ & 13.19 & 6164 & 3389 & 3087 & 208 & 152 \\
\hline 5 & Biomedical Sciences & 10.36 & 4845 & 2635 & 2385 & 35 & 45 \\
\hline 6 & Infectious Diseases & 3.52 & 1644 & 1023 & 652 & 10 & 8 \\
\hline 7 & Chemistry & 2.70 & 1260 & 623 & 656 & 8 & 6 \\
\hline 8 & $\begin{array}{l}\text { Economics, Politics and } \\
\text { Geography }\end{array}$ & 2.12 & 989 & 392 & 574 & 87 & 42 \\
\hline 9 & Clinical Medicine & 1.70 & 796 & 451 & 362 & 11 & 2 \\
\hline 10 & Social Studies & 1.54 & 720 & 427 & 313 & 12 & 16 \\
\hline 11 & Engineering & 1.32 & 615 & 336 & 281 & 5 & 4 \\
\hline 12 & Physics & 0.88 & 411 & 294 & 127 & 3 & 1 \\
\hline 13 & Health and Social Issues & 1.23 & 575 & 319 & 274 & 5 & 5 \\
\hline 14 & Materials Sciences & 1.10 & 512 & 319 & 219 & 11 & 2 \\
\hline 15 & Cognitive Sciences & 0.61 & 285 & 194 & 97 & 5 & 2 \\
\hline 16 & Psychology & 0.69 & 321 & 281 & 46 & 3 & 2 \\
\hline 17 & Computer Sciences & 0.46 & 215 & 132 & 89 & 6 & 5 \\
\hline \multirow[t]{2}{*}{18} & Business and Management & 0.31 & 143 & 63 & 83 & 3 & 2 \\
\hline & $\begin{array}{l}\text { Others (mainly arts \& } \\
\text { humanities) }\end{array}$ & 2.87 & 1339 & 869 & 484 & 13 & 28 \\
\hline
\end{tabular}

*Eighteen classes obtained after aggregation of WOS SCs (Rafols et al. 2014). Because of assignments to multiple WOS SCs to several classes, the total count $(57,288)$ for the whole region is 1.22 times higher than the actual count $(46,746)$.

mutual positions dictated by the global map of science based on aggregated journal citation data in 2015 (Rao, 1982; Rafols, 2010; Rafols et al., 2010; van Eck and Waltman, 2010; Leydesdorff, 2021). The colors (or gray shades) distinguish 18 classes (described below and listed in Table 4) identified as welldefined clusters in the global science map. The map in Fig. 3 was generated with customized VOSviewer parameters: repulsion $=0$, resolution $=4.5$, and the minimum cluster size $=6$. In the map, the WOS SCs forming the most representative clusters (Geosciences; Environmental Sciences; Plant Sciences; and Ecology) plot along the NNW to ESE swath (in between the regions occupied by Biomedical Sciences (left) and Engineering classes(right)). Several diversity indices measured by the dispersion of the HKHT publications over the different WOS SCs were as follows: (i) Rao-Stirling Diversity $(\Delta): 0.72$, and Leydesdorff's 
Div diversity (a measure roughly equivalent to $1-\Delta$ ): 0.15 , both scaled in between 0 to 1 , and (ii) Zhang's True Diversity 2D 3 : 1.6 , and Rousseau's Div* diversity: 32.8 , both obtained by multiplication by the number of subject categories and measured in an open scale (Leydesdorff et al., 2019; Rousseau, 2019; Stirling, 2007). These values suggest a relatively high degree of interdisciplinarity of the 1901-2018 HKHT publications. Such high interdisciplinarity, in publications retrieved through a search of database for keywords related only to regional terms for mountains and elevated plateau, points to the very close links of the human activity with the geographies.

\section{Focused Analysis of Research Publications in Field Sciences}

Prominence of the Field Science by Research Volume and its Impact

Field sciences delineated by WOS SCs were analyzed further (Table 5). Five SCs (Geosciences, Multidisciplinary; Geochemistry \& Geophysics; Meteorology \& Atmospheric Sciences; Geography, Physical; Geology) in Geosciences class and one each in other 3 classes (Environmental Sciences; Plant Sciences; and, Ecology) with more than 5\% share are targets of further analysis to reveal the significant trends. Considering the SCs coverage, relative completeness of abstracts in the database since 1991 as pointed by Ho (2013), quality of document types, access to indicators via InCites, and availability of some bibliometric studies for comparison (Gautam 2017; Gupta and Gupta, 2014;), further analysis is restricted to articles and reviews related to field sciences published during 1994-2018.

Publications data (document counts and shares) aggregated over 4 classes of field sciences using the 1984-2018 dataset are shown in Table 6. These data concern all documents and the Top $10 \%$ by citation documents discriminated by using the citation percentiles extracted from InCites. The average proportion of Top $10 \%$ papers for the field sciences is $13.1 \%$, which indicates a performance higher than the world average (10\%). Among the four classes, Geosciences with $16.1 \%$ value of PPTOP $10 \%$, clearly stands out and thus proves the relatively high citation impact of such research output from the HKHT region.

\section{Most Prolific Journals in Field Sciences}

Fifty journals dealing with the publications in field sciences and carrying 145-130 SCIE-indexed articles and reviews in field sciences at the level of the whole HKHT region are identified in Table 7. The rank of these journals determined by the number of papers for the whole region and the constituents is also given in

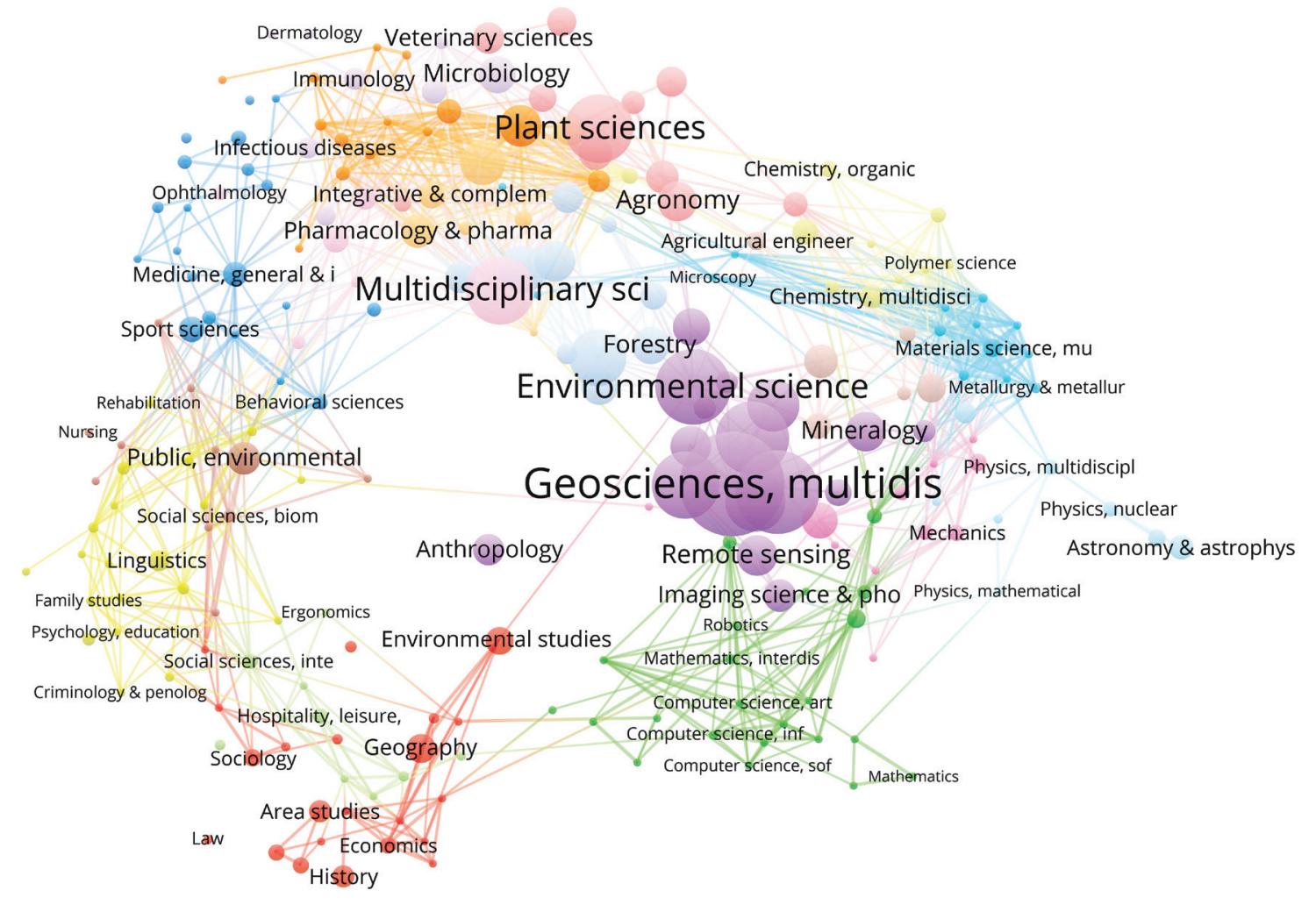

Fig. 3: A science overlay map for the HKHT region (period: 1901-2018). The most prolific WOS SCs, represented by the nodes (solid circles) scaled proportional to their counts, belong to four clusters (Geosciences, Environmental Sciences, Plant Sciences, and Ecology) situated along the NNW to ESE swath across the right side of the doughnutshaped map. 
Table 5: Breakdown of the HKHT research publications (1901-2018) into 4 field science disciplinary classes and related 39 WOS subject categories.

\begin{tabular}{|c|c|c|c|c|c|c|c|}
\hline \multirow[t]{2}{*}{ Field Sciences } & \multirow{2}{*}{ Web of Science Category } & \multicolumn{2}{|c|}{ HKHT region } & \multirow{2}{*}{$\begin{array}{l}\text { Tibet } \\
\text { Ndoc }\end{array}$} & \multirow{2}{*}{$\begin{array}{c}\text { Himalaya } \\
\text { Ndoc }\end{array}$} & \multirow{2}{*}{$\begin{array}{c}\text { Karakoram } \\
\text { Ndoc }\end{array}$} & \multirow{2}{*}{$\begin{array}{c}\text { Hindu Kush } \\
\text { Ndoc }\end{array}$} \\
\hline & & $\%$ share & Ndoc & & & & \\
\hline \multirow{12}{*}{ Geosciences } & Geosciences, Multidisciplinary & 22.64 & 10581 & 6298 & 5507 & 580 & 202 \\
\hline & Geochemistry \& Geophysics & 12.94 & 6047 & 4216 & 2914 & 250 & 172 \\
\hline & Meteorology \& Atmospheric Sciences & 8.84 & 4131 & 3101 & 1371 & 101 & 68 \\
\hline & Geography, Physical & 6.94 & 3242 & 2073 & 1496 & 263 & 92 \\
\hline & Geology & 5.09 & 2378 & 1531 & 1248 & 98 & 25 \\
\hline & Remote Sensing & 1.9 & 887 & 524 & 405 & 33 & 9 \\
\hline & Paleontology & 1.9 & 886 & 589 & 392 & 36 & 9 \\
\hline & Mineralogy & 1.63 & 763 & 542 & 327 & 20 & 4 \\
\hline & Imaging Sci \& Photogr Technology & 1.02 & 477 & 269 & 235 & 21 & 7 \\
\hline & Engineering, Geological & 0.63 & 295 & 77 & 216 & 11 & 6 \\
\hline & Engineering, Petroleum & 0.11 & 50 & 31 & 19 & 0 & 0 \\
\hline & Engineering, Ocean & 0.02 & 10 & 7 & 3 & 0 & 0 \\
\hline \multirow{6}{*}{$\begin{array}{l}\text { Environmental } \\
\text { Sciences \& } \\
\text { Technologies }\end{array}$} & Environmental Sciences & 9.47 & 4429 & 2587 & 2110 & 126 & 97 \\
\hline & Water Resources & 3.62 & 1694 & 753 & 1010 & 89 & 54 \\
\hline & Engineering, Civil & 1.28 & 598 & 389 & 220 & 22 & 14 \\
\hline & Engineering, Environmental & 1.13 & 527 & 395 & 148 & 6 & 1 \\
\hline & Limnology & 0.4 & 187 & 131 & 68 & 4 & 1 \\
\hline & Agricultural Engineering & 0.24 & 112 & 16 & 97 & 0 & 2 \\
\hline \multirow{11}{*}{$\begin{array}{l}\text { Agricultural } \\
\text { Sciences }\end{array}$} & Plant Sciences & 7.21 & 3370 & 1295 & 2203 & 31 & 37 \\
\hline & Agronomy & 1.71 & 798 & 301 & 506 & 1 & 2 \\
\hline & Soil Science & 1.51 & 704 & 435 & 272 & 3 & 2 \\
\hline & Agriculture, Multidisciplinary & 0.98 & 458 & 128 & 329 & 8 & 6 \\
\hline & Food Science \& Technology & 0.9 & 423 & 164 & 265 & 1 & 3 \\
\hline & Agriculture, Dairy \& Animal Science & 0.73 & 343 & 204 & 146 & 1 & 1 \\
\hline & Mycology & 0.68 & 320 & 94 & 237 & 2 & 0 \\
\hline & Integrative \& Complementary Medicine & 0.63 & 294 & 172 & 132 & 1 & 1 \\
\hline & Horticulture & 0.42 & 195 & 49 & 149 & 0 & 2 \\
\hline & Nutrition \& Dietetics & 0.22 & 102 & 52 & 53 & 0 & 1 \\
\hline & Materials Science, Paper \& Wood & 0.03 & 13 & 5 & 8 & 0 & 0 \\
\hline \multirow{10}{*}{$\begin{array}{l}\text { Ecological } \\
\text { Sciences }\end{array}$} & Ecology & 5.03 & 2349 & 1342 & 1111 & 29 & 26 \\
\hline & Zoology & 2.7 & 1261 & 711 & 618 & 14 & 10 \\
\hline & Evolutionary Biology & 1.98 & 926 & 662 & 375 & 1 & 2 \\
\hline & Forestry & 1.46 & 684 & 327 & 372 & 4 & 7 \\
\hline & Biodiversity Conservation & 1.44 & 674 & 327 & 381 & 14 & 15 \\
\hline & Entomology & 0.93 & 436 & 147 & 296 & 0 & 8 \\
\hline & Marine \& Freshwater Biology & 0.64 & 299 & 144 & 160 & 4 & 6 \\
\hline & Oceanography & 0.37 & 174 & 119 & 69 & 6 & 3 \\
\hline & Ornithology & 0.32 & 150 & 85 & 77 & 0 & 1 \\
\hline & Fisheries & 0.18 & 84 & 47 & 37 & 0 & 0 \\
\hline
\end{tabular}

Table 6: Document counts and shares of all $(43,595)$ and high impact (Top10\%: 4,561) publications in field science classes in the HKHT dataset for the 1994-2018 period.

\begin{tabular}{|c|c|c|c|c|c|}
\hline \multirow{2}{*}{ S. No. } & \multirow{2}{*}{ Field Sciences } & \multicolumn{2}{|c|}{ all (articles, reviews) } & \multicolumn{2}{|c|}{ Top $10 \%$ (articles, reviews) } \\
\hline & & Ndoc & share $(\%)$ & N(Top10\%) & $\begin{array}{l}\text { "PРТop } 10 \% \\
(\%)^{\prime}\end{array}$ \\
\hline 1 & Geosciences & $23,023.0$ & 65.1 & $3,712.0$ & 16.1 \\
\hline 2 & Environmental Sciences \& Technologies & $5,615.0$ & 15.9 & 392.0 & 7.0 \\
\hline 3 & Agricultural Sciences & $5,461.0$ & 15.5 & 474.0 & 8.7 \\
\hline \multirow[t]{2}{*}{4} & Ecological Sciences & $5,958.0$ & 16.9 & 651.0 & 10.9 \\
\hline & Field Sciences (1-4 above, no duplicates) & 35,344 & 81.1 & 4,561 & 13.1 \\
\hline
\end{tabular}

${ }^{*}$ PPTop $10 \%$ is the proportion of number of documents in top $10 \%$ category (NTop10\%) with respect to all papers (Ndoc) by citation. 
the respective columns. For ease of understanding, the top 10 most popular journals, in abbreviated forms, for the whole region and 5 journals for its constituents are presented below in the order of decreasing document counts.

HKHT region: J ASIAN EARTH SCI; ACTA PETR SIN; EARTH PLANET SCI LETT; CHINESE J GEOPHYS-CHINESE ED; TECTONOPHYSICS; CURR SCI; J GEOL SOC INDIA; J GEOPHYS RES-ATMOS; J GEOPHYS RES-SOLID EARTH; GEOPHYS RES LETT

Tibet: ACTA PETR SIN; CHINESE J GEOPHYSCHINESE ED; J ASIAN EARTH SCI; EARTH PLANET SCI LETT; TECTONOPHYSICS

Himalaya: CURR SCI; J GEOL SOC INDIA; J ASIAN EARTH SCI; EARTH PLANET SCI LETT; TECTONOPHYSICS

Karakoram: EARTH PLANET SCI LETT; TECTONICS; J ASIAN EARTH SCI; GEOMORPHOLOGY; GEOL SOC AMER BULL

Hindukush: MT RES DEV; PAKISTAN J BOT; EARTH PLANET SCI LETT; J ASIAN EARTH SCI; J GEOPHYS RES-SOLID EARTH

Two international and relatively high impact journals (Journal of Asian Earth Sciences; Earth and Planetary Science Letters) are popular in all subsets. In Tibet and Himalaya subsets, domestic journals published in India and China, the top producers, occupy the top positions by document counts. Mountain Research and Development, an international journal specifically devoted to sustainable development in the world's mountains, ranks 1 st as a publication source on Hindukush but 8th and 11th on Karakoram and Himalaya, respectively. Judging from the journal impact factor (JIF), an indicator of the degree of citation of a journal, the top two journals with the highest document counts in each of the 3 regions (Tibet, Himalaya and Hindukush) have a relatively low impact (JIF for 2018: 0.8-1.4).

\section{Characterization of High Impact (Top 10\%) Publications in Field Sciences}

Science mapping techniques are used to analyze a subset of 4,561 high impact 1994-2018 HKHT papers (those belonging to the top $10 \%$, among the WOSrecorded publications of the same WOS SC and publication year, by citation) in field sciences. These are aimed at identifying the most prolific entities (e.g., journals, fields, and words/phrases representative of the most important research themes) associated with the high impact publications, also enabling a comparison of the results with those obtained from the complete dataset for the whole region.

Figure 4 shows a science map of WOS SCs related to TOP $10 \%$ documents in field sciences. The size of each node (filled circle) is proportional to the number of documents assigned to the SC by whole counting, while the node color (or gray shade) identifies specific cluster defined by the co-occurring $\mathrm{SCs}$. The connecting line thickness is proportional to the strength of links between nodes. Seven SCs (Geosciences, Multidisciplinary; Geochemistry \& Geophysics; Meteorology \& Atmospheric Sciences; Geography, Physical; Environmental Sciences; Ecology and Plant Sciences) lead the SC clusters.

Bibliographic coupling of sources, journals in which 30 or more TOP $10 \%$ documents were published, is

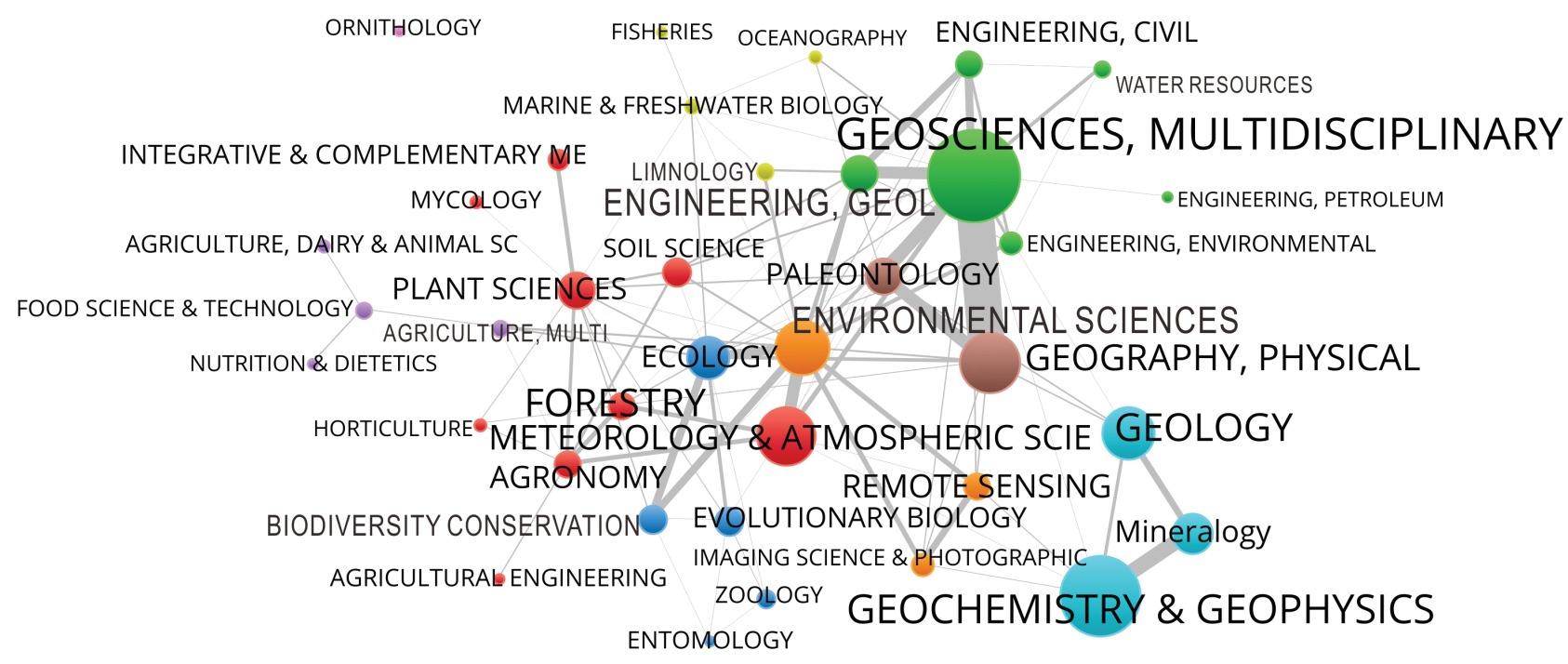

Fig. 4: A subject-based science map depicting the co-occurrences of field science categories represented by 4,561 TOP $10 \%$ documents from the HKHT region. 
used to generate the map shown in Figure 5. Eleven journals, each carrying TOP $10 \%$ documents ranging from 280 to 79 (Earth Planet Sci Lett; Geology; Paleogeogr Paleoclimatol Paleoecol; Gondwana Res; J Geophys Res-Solid Earth; Lithos; J Asian Earth Sci; Geophys Res Let; Geol Soc Amer Bull, Tectonics; Tectonophysics) are remarkable. Among these high impact sources, five journals (J Asian Earth Sci; Earth Sci Planet Lett; Tectonophysics; Geophys Res Lett; J Geophys Res-Solid Earth) are found to be the most productive in terms of publication counts in field sciences from the HKHT region (Table 7).

A science map depicting the co-occurrence of all keywords limited to noun phrases comprising at least 2 words in 4,561 documents is shown in Figure 6. From the map and underlying cluster-related data, the following prolific as well as high impact themes (each represented by 4 major key-phrases and occupying approximate map position (in Fig. 6) shown within the brackets) have been identified:

1) climate change; mass balance; black carbon; remote sensing (lower right)

2) tectonic evolution; continental collision; volcanic rock; u-pb geochronology (lower left)

3) india asia collision; lower crustal flow; active tectonics; crustal structure (upper right)

4) stable isotope; environmental change; asian summer monsoon; ice core (upper middle)

5) main central thrust; inverted metamorphism; channel flow; shear zone (lower middle)

6) chloroplast dna; medicinal plants;molecular phylogeny; genetic diversity (upper right)

Themes 1) and 4) are related to environmental/climatic changes, while themes 2) and 3) are associated with the India-Asia collision tectonics, volcanism, mountain building processes and their timing and thus serve as the targets of continued attention from the HKHT research community to the. Theme 5) is related to the major thrust system extending throughout the $>2,500$ $\mathrm{km}$ length of the Himalayan arc and associated with it inverted metamorphism, shearing and channel flow process within the Earth's crust.

The sixth cluster is related to the study of genetic diversity including those of the medicinal plants. The prominence of the research theme reflecting this cluster is consistent with the findings of Hao et al. (2019) derived from a study of 1998-2017 documents retrieved using a set of strings (variants of plant, flora and botany) representing "plants" and "Himalaya". Hao et al. (2019) noted the importance of the research on Himalayan plants (application of the compounds derived from them in clinical medicine, pharmacology and oncology mediated by research on chemistry, genetics, and microbiology) in the 21st century and highlighted the divergence of research on ethnopharmacology into (a) phytochemistry \& biotechnology, and (b) medicinal biodiversity and conservation of traditional ethnobotanical knowledge.

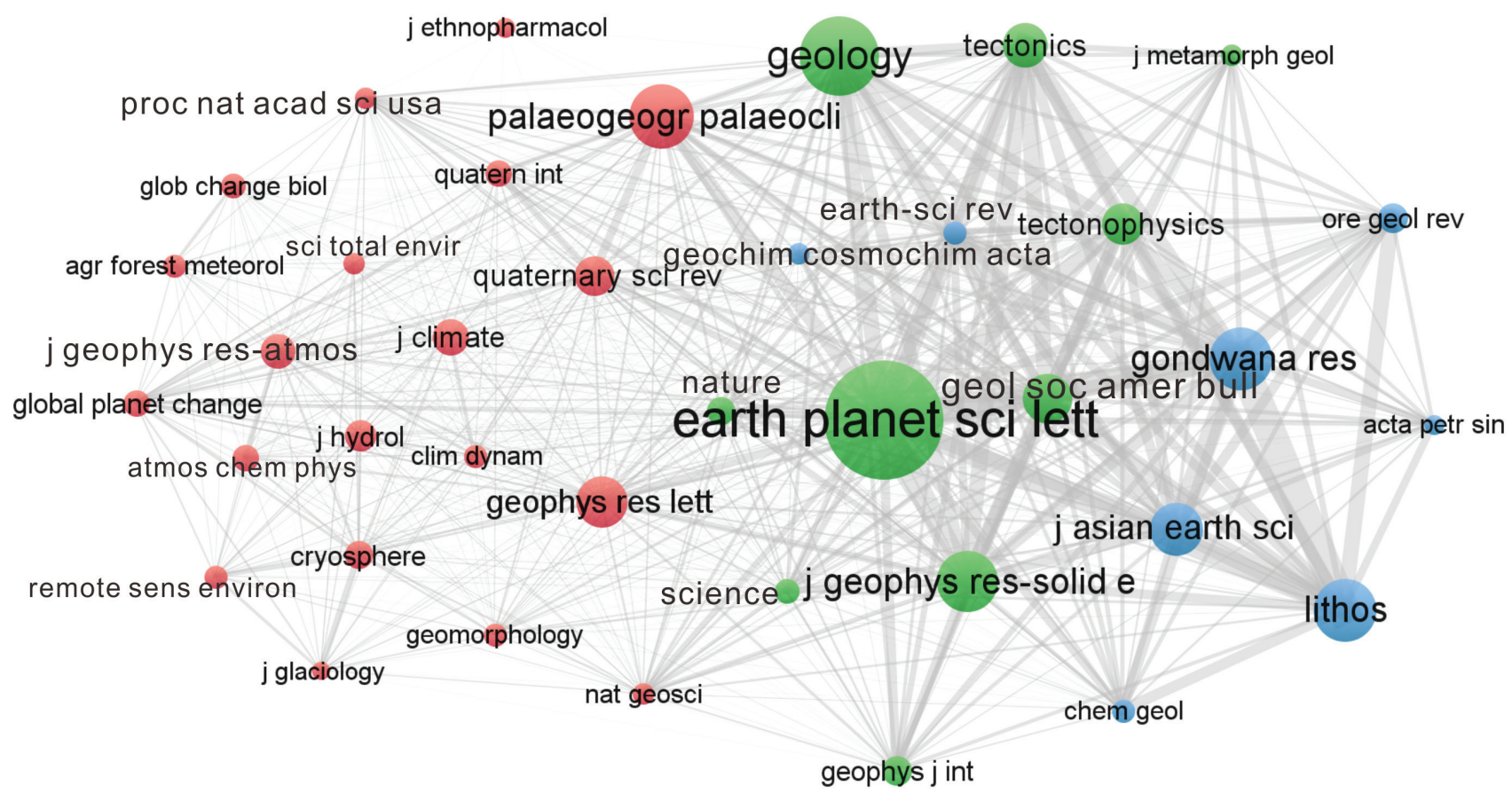

Fig. 5: Bibliographic coupling of journals in which the high impact (TOP10\% by citation papers) in fields sciences related to the HKHT region were published. Each journal plotted here has at least 30 documents. 
Table 7: Top 50 journals titles in field sciences related to 1994-2018 publications from the HKHT region.

\begin{tabular}{|c|c|c|c|c|c|c|c|}
\hline \multirow{2}{*}{$\begin{array}{l}\text { Rank } \\
\text { HKHT }\end{array}$} & \multirow{2}{*}{ Journal Name } & \multirow{2}{*}{$\begin{array}{l}\mathrm{N}(\text { art, rev) } \\
\text { HKHT }\end{array}$} & \multirow{2}{*}{$\begin{array}{l}\text { Share, \% } \\
\text { HKHT }\end{array}$} & \multicolumn{4}{|c|}{ Journal Rank } \\
\hline & & & & Tibet & Himalaya & Karakoram & Hindu Kush \\
\hline 1 & JOURNAL OF ASIAN EARTH SCIENCES & 830 & 2.39 & 3 & 3 & 3 & 4 \\
\hline 2 & ACTA PETROLOGICA SINICA & 675 & 1.95 & 1 & 7 & 30 & 35 \\
\hline 3 & EARTH AND PLANETARY SCIENCE LETTERS & 633 & 1.82 & 4 & 4 & 1 & 3 \\
\hline 4 & $\begin{array}{l}\text { CHINESE JOURNAL OF GEOPHYSICS-CHINESE } \\
\text { EDITION }\end{array}$ & 619 & 1.78 & 2 & 29 & 24 & 12 \\
\hline 5 & TECTONOPHYSICS & 573 & 1.65 & 5 & 5 & 7 & 8 \\
\hline 6 & CURRENT SCIENCE & 538 & 1.55 & 102 & 1 & 10 & 25 \\
\hline 7 & JOURNAL OF THE GEOLOGICAL SOCIETY OF INDIA & 449 & 1.29 & 68 & 2 & 16 & 88 \\
\hline 8 & $\begin{array}{l}\text { JOURNAL OF GEOPHYSICAL RESEARCH- } \\
\text { ATMOSPHERES }\end{array}$ & 449 & 1.29 & 6 & 24 & 27 & 20 \\
\hline 9 & $\begin{array}{l}\text { JOURNAL OF GEOPHYSICAL RESEARCH-SOLID } \\
\text { EARTH }\end{array}$ & 441 & 1.27 & 7 & 9 & 11 & 5 \\
\hline 10 & GEOPHYSICAL RESEARCH LETTERS & 397 & 1.14 & 13 & 14 & 13 & 6 \\
\hline 11 & QUATERNARY INTERNATIONAL & 394 & 1.14 & 12 & 18 & 14 & 21 \\
\hline 12 & ACTA GEOLOGICA SINICA-ENGLISH EDITION & 377 & 1.09 & 8 & 38 & 38 & 89 \\
\hline 13 & $\begin{array}{l}\text { PALAEOGEOGRAPHY PALAEOCLIMATOLOGY } \\
\text { PALAEOECOLOGY }\end{array}$ & 369 & 1.06 & 9 & 27 & 39 & n.a. \\
\hline 14 & LITHOS & 338 & 0.97 & 14 & 23 & 20 & 90 \\
\hline 15 & TECTONICS & 326 & 0.94 & 15 & 13 & 2 & 10 \\
\hline 16 & SCIENCE IN CHINA SERIES D-EARTH SCIENCES & 323 & 0.93 & 11 & 59 & 101 & n.a. \\
\hline 17 & CHINESE SCIENCE BULLETIN & 323 & 0.93 & 10 & 51 & 102 & n.a. \\
\hline 18 & GEOPHYSICAL JOURNAL INTERNATIONAL & 321 & 0.92 & 16 & 21 & 31 & 7 \\
\hline 19 & GEOLOGY & 321 & 0.92 & 24 & 8 & 17 & 36 \\
\hline 20 & ZOOTAXA & 310 & 0.89 & 28 & 19 & 137 & 37 \\
\hline 21 & GONDWANA RESEARCH & 309 & 0.89 & 18 & 17 & 25 & 17 \\
\hline 22 & JOURNAL OF MOUNTAIN SCIENCE & 297 & 0.86 & 32 & 16 & 15 & 9 \\
\hline 23 & PLOS ONE & 288 & 0.83 & 21 & 30 & 70 & 38 \\
\hline 24 & ENVIRONMENTAL EARTH SCIENCES & 279 & 0.8 & 29 & 22 & 40 & 53 \\
\hline 25 & GEOMORPHOLOGY & 271 & 0.78 & 39 & 10 & 4 & 13 \\
\hline 26 & JOURNAL OF CLIMATE & 261 & 0.75 & 17 & 92 & 79 & 54 \\
\hline 27 & INTERNATIONAL JOURNAL OF CLIMATOLOGY & 259 & 0.75 & 23 & 49 & 48 & 22 \\
\hline 28 & SCIENTIFIC REPORTS & 249 & 0.72 & 20 & 67 & 34 & 55 \\
\hline 29 & MOUNTAIN RESEARCH AND DEVELOPMENT & 237 & 0.68 & 75 & 11 & 8 & 1 \\
\hline 30 & SCIENCE CHINA-EARTH SCIENCES & 236 & 0.68 & 19 & 80 & 138 & 39 \\
\hline 31 & HIMALAYAN GEOLOGY & 228 & 0.66 & 196 & 6 & 35 & 40 \\
\hline 32 & CLIMATE DYNAMICS & 224 & 0.65 & 26 & 47 & 18 & 18 \\
\hline 33 & NATURAL HAZARDS & 221 & 0.64 & 107 & 12 & 49 & 14 \\
\hline 34 & SCIENCE OF THE TOTAL ENVIRONMENT & 217 & 0.63 & 30 & 53 & 36 & 29 \\
\hline 35 & QUATERNARY SCIENCE REVIEWS & 216 & 0.62 & 27 & 39 & 12 & n.a. \\
\hline 36 & ADVANCES IN ATMOSPHERIC SCIENCES & 215 & 0.62 & 22 & 244 & n.a. & n.a. \\
\hline 37 & COLD REGIONS SCIENCE AND TECHNOLOGY & 206 & 0.59 & 25 & 290 & 139 & n.a. \\
\hline 38 & REMOTE SENSING & 193 & 0.56 & 31 & 128 & 41 & n.a. \\
\hline 39 & GLOBAL AND PLANETARY CHANGE & 193 & 0.56 & 33 & 57 & 28 & 19 \\
\hline 40 & GEOLOGICAL SOCIETY OF AMERICA BULLETIN & 189 & 0.54 & 35 & 25 & 5 & 56 \\
\hline 41 & ATMOSPHERIC CHEMISTRY AND PHYSICS & 184 & 0.53 & 34 & 41 & 71 & 57 \\
\hline 42 & $\begin{array}{l}\text { BULLETIN OF THE SEISMOLOGICAL SOCIETY OF } \\
\text { AMERICA }\end{array}$ & 173 & 0.5 & 41 & 40 & 140 & 15 \\
\hline 43 & JOURNAL OF EARTH SYSTEM SCIENCE & 171 & 0.49 & 163 & 15 & 57 & 58 \\
\hline 44 & INTERNATIONAL JOURNAL OF REMOTE SENSING & 171 & 0.49 & 51 & 28 & 50 & 59 \\
\hline 45 & THEORETICAL AND APPLIED CLIMATOLOGY & 160 & 0.46 & 36 & 79 & 51 & 91 \\
\hline 46 & JOURNAL OF HYDROLOGY & 160 & 0.46 & 48 & 37 & 22 & 11 \\
\hline 47 & ATMOSPHERIC ENVIRONMENT & 153 & 0.44 & 42 & 50 & 141 & 60 \\
\hline 48 & CHEMICAL GEOLOGY & 149 & 0.43 & 49 & 33 & 80 & n.a. \\
\hline 49 & INTERNATIONAL GEOLOGY REVIEW & 147 & 0.42 & 37 & 72 & 52 & 92 \\
\hline 50 & PAKISTAN JOURNAL OF BOTANY & 145 & 0.42 & 199 & 26 & 23 & 2 \\
\hline
\end{tabular}

$\mathrm{N}($ art, rev) and share (\%) in 3rd and 4th columns are related to the whole HKHT region. Journal rank is determined by the publication volume (number of documents) in each region. 


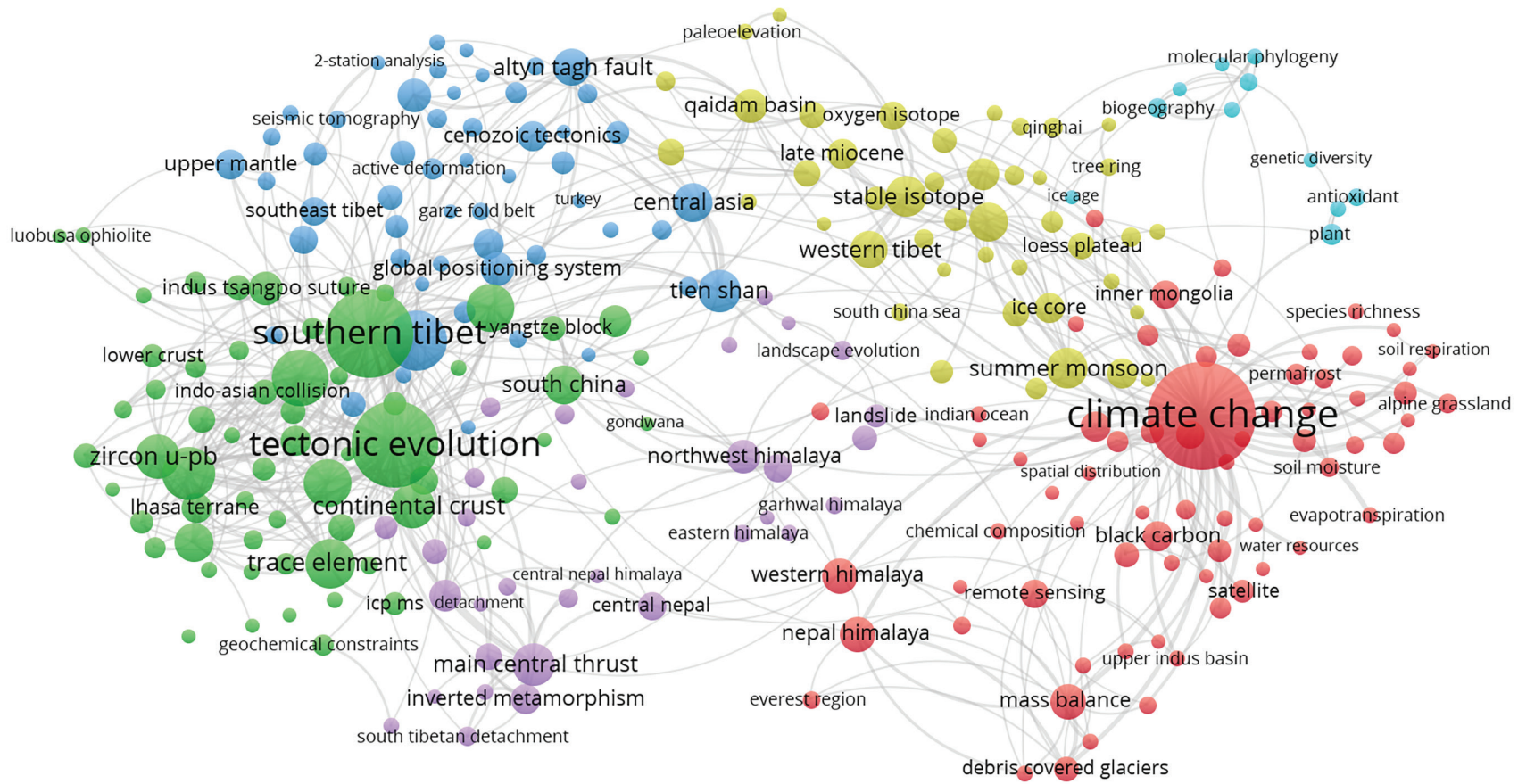

Fig. 6: A VOSviewer science map generated from noun phrases comprising all kinds of keywords related to 4,561 TOP10\% documents in field sciences related to the HKHT region. For clarity, singular words including those for the constituents of the HKHT region were excluded.

\section{DISCUSSION}

This reconnaissance bibliometric survey covering the period from 1901 to 2018 suggests the whole HKHT region with all its constituent mountain ranges and plateau to be a continuing research target of research communities from countries both internal and external to the region. Less than $2 \%$ of the recorded citable items appeared before 1980, a gradual increase followed thereafter for about a decade, and finally an accelerated and exponential growth began in the last decade of the 20th century. These publications are the result of the large-scale research efforts from countries directly related to the HKHT region as well as the concentrated international efforts from academia, governments and international not-forprofit organizations dedicated to better understanding the mountain-building and monsoon regime, climate change and natural disasters (earthquakes, floods, landslides), formation of collaborative research forums or teams including the local research communities, and identification of common issues and possible solutions for integrated and sustainable development of this very large mountain system. These efforts are well reflected in the research publications as evident from the prominence of field sciences (represented mainly by earth, environmental and agricultural sciences). A highly collaborative and cross-disciplinary research characterizes the HKHT region as shown by the diversity in entities (countries, institutions, and research organizations) related to high impact publications.

Due to the reconnaissance nature of this study, the few research themes characterized by both in quantity (large volume) and quality (impact) identified here are related to mainly earth and environmental processes characteristic of the whole HKHT region. As the field research activities in specific time period by individual or group of international researchers might have been concentrated to specific constituent/segment of the HKTH mountain and plateau region, some of them receiving greater attention than the others (e.g., Searle 2013), the significance of these themes and the spatial and temporal extent within the region need to be detailed further through studies restricted to smaller geographic regions.

In the case of Himalaya, for example, there are several East-West extending geotectonic belts (i.e., Indo-Ganga Plain $\rightarrow$ Sub Himalaya $\rightarrow$ Lesser Himalaya $\rightarrow$ Higher Himalaya $\rightarrow$ Tibetan Tethys, from South to North) (Dhital 2015; Yin 2006). These belts, each of which has characteristic but distinct topography, geomorphological maturity, tectonics/structure, geological composition \& age span, and varies in length up to about $2,500 \mathrm{~km}$ and in width up to tens of kilometers, are separated by major structural 
discontinuities (faults or thrusts) such as the Himalayan Frontal Thrust, Main Boundary Fault/Thrust, Main Central Thrust, South Tibetan Detachment Thrust and the Indus Tsangpo Suture that forms the northernmost margin of the Himalaya in geologic terms. All these major faults or thrusts, dipping towards North at various angles, are connected with a relatively lowangle and north-dipping trunk thrust called the Main Himalayan Thrust, rupture along which gives rise to medium to large-sized, often devastating to life and infrastructure, earthquakes like the 7.8 magnitude Gorkha Earthquake of 2015 in central Nepal (Miyake et al. 2017). To reveal the prominent research themes and entities in Himalaya, therefore, it will be necessary to breakdown the research publications into subsets in space (constituent region, geotectonic belts or specific elements such as thrust zones) and time (publication period) using an objective strategy and then analyze/ map the subsets separately.

This study provided a brief summary of the HKHT research publications in terms of a few research entities: constituent regions, countries, organizations, subject areas or disciplinary classes, and journals for the whole dataset and additionally identified the most prolific themes evident from the relatively highly cited subset related to field sciences. Because of the reconnaissance nature, the rather large scale of the region as well as the dataset (period covered, number of publications), and lack of author disambiguation data, no attempt was made to analyze data at the author level. So, direct comparison of these data with results of previous studies limited to selected countries/ regions/geographic or disciplinary targets (Gupta 2011; Liu et al. 2012; Prasad et al. 2016; Gautam 2017; Wang 2017; Emmer 2018; Hao et al. 2019) is not possible. However, the major conclusions derived in this study, especially related to the prominence of field sciences, prolific themes, prominent journals, topmost contributors are in agreement with those studies. The data presented here are believed to be of importance for a variety of global users (researchers, students, universities and research organizations as well as other stakeholders (e.g., governments, funding organizations, non-profit organizations) interested in the HKHT region. These data can be used for planning further detailed analyses on targets (e.g., specific geographic regions, disciplinary areas, other research entities such as institutions or research teams) considering the space and time factors.

As a WOS-based study based on documents (articles, reviews, Letters and Notes) in journals that meet strict selection criteria, the outcome and inferences presented here generally reflect the most globally visible research related to the HKHT region. Concerns raised elsewhere (e.g., Gautam 2017) suggest that inclusion of other types of documents such as conferene papers and book chapters, even if restricted to the WOS database, and whenever possible consulting other databases (e.g., Scopus) with better coverage of regional journals including those originating from the countries in the HKHT region, will provide better insights and more representative picture on the research landscape of such a vast region. A broader set of publications, selected using reasonable quality criteria for their sources, could be a justified target for detailed bibliometric/scientometric analysis other than the impact based on the citation data known to largely vary across databases.

\section{CONCLUSIONS}

Majors findings of the preliminary bibliometric survey and science mapping of the WOS-based research publications on the HKHT region are as follows:

(1) The HKHT publications for 1901-2018 period are represented by 46,746 citable items (articles, reviews, letters and notes) with $98 \%$ postdating 1980. With 244 WOS subject categories, they exhibit high interdisciplinarity and international character ( 79 countries with 15 or more documents). While China and India, native to the region, top the list of contributors, USA, Germany, England, France, Japan, Australia, and Canada follow as the largest external contributors. Nepal and Pakistan, native to the HKHT region, occupy the 10th and 11th positions, respectively.

(2) Nine subject categories related to "earth, environmental and agricultural sciences" exhibit highest shares $(22.6 \%$ to $3.2 \%$ of the total publications) with the prominence of 4 broader disciplinary classes (Geosciences, Environmental Sciences \& Technologies, Agricultural Sciences, and Ecological Sciences) attributed to "field sciences". The average proportion of high impact (Top10\%) documents for the field sciences is $13.1 \%$ implying that the research impact is higher than the world average (10\%). Five international journals (J Asian Earth Sci; Earth Sci Planet Lett; Tectonophysics; Geophys Res Lett; J Geophys Res-Solid Earth) published outside the HKHT region are recognized to be the best in terms of the citation impact and the publication volume.

(3) Science mapping reveals five high impact themes (climate change including monsoon regime, tectonic evolution of the mountain orogen, IndiaAsia collision and associated crustal phenomena, activities on major thrusts, channel flows, and inverted metamorphism) related to earth sciences, and a sixth theme dealing with the genetic diversity of plants, mainly of the medicinal values.

Apart from these notable findings, this study identified several characteristics (i.e., the most prolific research institutions, the disciplinary structure, and 
the publication sources) of the individual constituent regions (Himalaya, Tibet, Karakoram, Hindukush). The outcome of this study is believed to serve as the basis of further detailed research for targets as well as a variety of global users (researchers, students, universities and research organizations and other local, regional and global stakeholders engaged in and keeping interest in the HKHT region.

\section{ACKNOWLEDGEMENT}

This research was accomplished using the facilities at the Research Development Section, under the MEXTfunded Program for Enhancing Institutional Capacity, Hokkaido University, Sapporo, Japan.

\section{REFERENCES}

Aksnes D.W., and Sivertsen, G., 2019, A Criteria-based Assessment of the Coverage of Scopus and Web of Science. Journal of Data and Information Science, 4(1), pp. 1-21.

Clarivate Analytics, 2019, https://clarivate.com/ webofsciencegroup/. Web of Science and InCites, accessed May 28, 2019.

Dhital M.R., 2015, Geology of the Nepal Himalaya: Regional Perspective of the Classic Collided Orogeny. Springer International Publishing, 498pp.

Emmer, A., 2018, GLOFs in the WOS: bibliometrics, geographies and global trends of research on glacial lake outburst floods (Web of Science, 1979-2016). Natural Hazards and Earth System Science, 18, pp. 813-827.

Gautam, P., Watanabe, T., Nishi, H., and Yasunari, T., 2005, Preface, Thematic Section A: Uplift of the HimalayaTibet region and the Asian monsoon. The Island Arc, 14, pp. 1-2.

Gautam, P., 2017, An overview of the Web of Science record of scientific publications (2004-2013) from Nepal: focus on disciplinary diversity and international collaboration. Scientometrics, 113, pp. 1245-1267.

Gautam, P., 2019, A Bibliometric Approach for DepartmentLevel Disciplinary Analysis and Science Mapping of Research Output Using Multiple Classification Schemes. Journal of Contemporary Eastern Asia, 18(1), pp. 7-29. DOI: 10.17477/jcea.2019.18.1.007

Glänzel, W., 2003, A Course on Theory and Application of Bibliometric Indicators, Course Handouts. https:// www.researchgate.net/publication/242406991 (accessed on 2016/8/18).

Gupta, B.M., 2011, Ranking of Indian institutions in agriculture \& allied sciences for their research output during 1999-2008. Annals of Library and Information Studies, 58, pp. 63-70.

Gupta, B.M., and Gupta, R., 2014, A Scientometric Assessment of Indian Himalayan R\&D Publications during 2004-13. Library Philosophy and Practice (e-journal), 1203. http://digitalcommons.unl.edu/

\section{libphilprac/1203}

Hao, Da-Cheng, Yuan, Xue, Di, Yan-Hong, and Xiao, PeiGen, 2019, Global Himalaya plant research trend and performance in science citation index from 1998 to 2017. Pakistan Journal of Botany, 51. 10.30848/ PJB2019-5(26).

Ho, Y.S., 2013, Comments on "a bibliometric study of earthquake research: 1900-2010". Scientometrics, 96(3), p. 929. https://doi.org/10.1007/s11192-0120915-2

Kandel, P., Chettri, N., Chaudhary, S., Sharma, P., and Uddin, K., 2021, Ecosystem services research trends in the water tower of Asia: A bibliometric analysis from the Hindu Kush Himalaya, Ecological Indicators, 121, 107152. https://doi.org/10.1016/j. ecolind.2020.107152.

Leydesdorff, L., 2021, A method for generating overlay maps on the basis of aggregated journal-journal citation relations in 2015. http://www.leydesdorff.net/ wc15/index.htm (accessed January 30, 2021).

Leydesdorff, L., Wagner, C.S., and Bornmann, L., 2019, Interdisciplinarity as Diversity in Citation Patterns among Journals: Rao-Stirling Diversity, Relative Variety, and the Gini coefficient. Journal of Informetrics, 13(1), pp. 255-264.

Liu, X., Zhan, F.B., Hong, S. et al., 2012, A bibliometric study of earthquake research: 1900-2010. Scientometrics, 92(3), pp. 747-765. https://doi.org/10.1007/s11192011-0599-z

Miyake, H., Sapkota, S.N., Upreti, B.N. et al., 2017, Special issue "The 2015 Gorkha, Nepal, earthquake and Himalayan studies: First results". Earth Planets Space, 69(12). https://doi.org/10.1186/s40623-016$0597-8$

Prasad, R.C., Pande, G.P., Singh, R.K., and Prasad, R., 2016, Scientometrics Exploration of Research Publications on Himalayas during the Year 1989-2014: A Scientific Review. International Journal of Basic and Applied Sciences, 5(3), pp. 102-108.

Rafols, I., 2014, Knowledge Integration and Diffusion: Measures and Mapping of Diversity and Coherence. In: Ding Y., Rousseau R., Wolfram D. (eds) Measuring Scholarly Impact, Springer, Cham, pp. 169-190.

Rafols, I., Porter, A.L., and Leydesdorff, L., 2010, Science overlay maps: a new tool for research policy and library management. Journal of the American Society for Information Science \& Technology, 61(9), pp. 1871-1887.

Rao, C.R., 1982, Diversity: Its measurement, decomposition, apportionment and analysis. Sankhya: The Indian Journal of Statistics, Series A, 44(1), pp. 1-22.

Rousseau, R., 2019, On the Leydesdorff-WagnerBornmann proposal for diversity measurement. Journal of Informetrics, 13(3), pp. 906-907. https:// doi.org/10.1016/j.joi.2019.03.015

Searle, M., 2013, Colliding Continents: a geological exploration of the Himalaya, Karakoram, and Tibet. 
Oxford University Press, 444p.

Stirling, A., 2007, A general framework for analysing diversity in science, technology and society. Journal of the Royal Society Interface, 4(15), pp. 707719.

van Eck, N.J., and Waltman, L., 2010, Software survey: VOSviewer, a computer program for bibliometric mapping. Scientometrics, 84(2), 523-538.

Wang, T., 2017. A bibliometric analysis of international research on Tibetan Plateau: Based on the databases of SCIE and ESI. Progress in Geography, 36(4), 500-
512. DOI: 10.18306/dlkxjz.2017.04.011 (in Chinese, with English Abstract)

Wester, O., Mishra, A., Mukherji, A., and Shrestha, A.B. (eds), 2019, The Hindu Kush Himalaya Assessment-Mountains, Climate Change, Sustainability and People, Springer Nature Switzerland AG, Cham.

Yin, A., 2006, Cenozoic tectonic evolution of the Himalayan orogen as constrained by along-strike variation of structural geometry, exhumation history, and foreland sedimentation. Earth-science Reviews, 76 (1-2), pp. $1-131$. 